Instituto Internacional de Investigación y Desarrollo Tecnológico Educativo INDTEC, C.A.

DOI: https://doi.org/10.29394/Scientific.issn.2542-2987.2020.5.18.4.85-107

OAI-PMH: http://www.indteca.com/ojs/index.php/Revista Scientific/oai

Artículo Original / Original Article

\title{
Educación a distancia en las universidades venezolanas ante la pandemia COVID-19: Desafíos y Oportunidades
}

\author{
Autoras: Eddymar María Flores Nessi \\ Universidad Politécnica Territorial del Zulia, UPTZ \\ eddymarf.nessi@gmail.com \\ Zulia, Venezuela \\ https://orcid.org/0000-0003-1786-2283 \\ Jenny Macarena Meléndez Mora \\ Universidad Politécnica Territorial del Zulia, UPTZ \\ immelendezm@gmail.com \\ Zulia, Venezuela \\ https://orcid.org/0000-0002-2802-1159 \\ María Alejandra Baptista Montero \\ Universidad Bolivariana de Venezuela, UBV \\ marialebap@gmail.com \\ Zulia, Venezuela \\ https://orcid.org/0000-0002-1761-8929
}

\section{Resumen}

El propósito de este artículo es describir la experiencia de implementar la Educación a Distancia adoptada ante la emergencia sanitaria provocada por la pandemia COVID-19. Se analiza la experiencia de la Universidad Politécnica Territorial del Zulia (UPTZ) con el fin de reflexionar sobre desafíos y oportunidades que enfrentan las universidades ante la virtualización de la enseñanza; cuestionando si los docentes y estudiantes disponen de herramientas tecnológicas, así como la adecuada formación en entornos virtuales para el desarrollo de las actividades académicas y de investigación. Se fundamentó en los planteamientos de Juca (2016); Lozano y Giralt (2014); Flores y Garrido (2019); Ramírez (2016); entre otros. La metodología es de tipo descriptiva, teniendo en cuenta que sigue tanto el enfoque cualitativo como el cuantitativo; se utilizaron diversas técnicas e instrumentos como registros observacionales, la revisión documental; y se aplicó un instrumento de evaluación diagnóstica a 27 docentes y 15 estudiantes. Las ideas generadas nacen como resultado de un proceso de reflexión que se ha venido dando en el contexto actual del Plan Universidad en Casa ante limitaciones de conectividad virtual; reconociendo el esfuerzo y corresponsabilidad de la comunidad académica para llevar a cabo las actividades bajo la modalidad a distancia.

Palabras clave: educación a distancia; tecnología; comunicación; universidad.

Cómo citar este artículo:

Flores, E., Meléndez, J., \& Baptista, M. (2020). Educación a distancia en las universidades venezolanas ante la pandemia COVID-19: Desafíos y Oportunidades. Revista Scientific, 5(18), 85107, e-ISSN: 2542-2987. Recuperado de: https://doi.org/10.29394/Scientific.issn.2542$\underline{\underline{2987.2020 .5 .18 .4 .85-107}}$

Fecha de Recepción: 12-06-2020
Fecha de Aceptación: 10-09-2020
Fecha de Publicación: 05-11-2020 
OAI-PMH: http://www.indteca.com/ojs/index.php/Revista_Scientific/oai

Artículo Original / Original Article

\title{
Distance education in Venezuelan universities in the face of the COVID- 19 pandemic: Challenges and Opportunities
}

\begin{abstract}
The purpose of this article is to describe the experience of implementing Distance Education adopted in the face of the health emergency caused by the COVID-19 pandemic. The experience of the Territorial Polytechnic University of Zulia (UPTZ) is analyzed in order to reflect on the challenges and opportunities faced by universities in the face of virtualization of teaching; questioning whether teachers and students have technological tools, as well as adequate training in virtual environments for the development of academic and research activities. It was based on the proposals of Juca (2016); Lozano and Giralt (2014); Flores and Garrido (2019); Ramírez (2016); among others. The methodology is descriptive, taking into account that it follows both the qualitative and the quantitative approach; Various techniques and instruments were used such as observational records, documentary review; and a diagnostic evaluation instrument was applied to 27 teachers and 15 students. The ideas generated are born as a result of a process of reflection that has been taking place in the current context of the Home University Plan in the face of virtual connectivity limitations; recognizing the effort and co-responsibility of the academic community to carry out activities under the distance modality.
\end{abstract}

Keywords: distance education; technology; communication; university.

How to cite this article:
Flores, E., Meléndez, J., \& Baptista, M. (2020). Distance education in Venezuelan universities in
the face of the COVID-19 pandemic: Challenges and Opportunities. Revista Scientific, 5(18), 85-
$\begin{array}{ll}107, \quad \text { e-ISSN: 2542-2987. Recovered from: https://doi.org/10.29394/Scientific.issn.2542- } \\ 2987.2020 .5 .18 .4 .85-107\end{array}$

Date Received:

12-06-2020
Date Acceptance:

10-09-2020
Date Publication:

05-11-2020 


\section{Introducción}

La emergencia sanitaria causada por la pandemia COVID-19 ha impactado el desarrollo actividades administrativas, académicas y de investigación en las universidades desde el pasado 15 de marzo del año 2020, fecha en la cual se decreta en Venezuela el Estado de Alarma que dispuso la suspensión de actividades presenciales dentro de las instituciones educativas. A partir de ese entonces, el Ministerio de Popular para la Educación Universitaria giro instrucciones a las diferentes instituciones universitarias de gestión pública y/o privada a implementar un Plan de Prosecución de estudios a distancia, denominado "Universidad en Casa", con el fin de mantener las actividades de formación académica.

En atención a las medidas preventivas tomadas por la aparición de los primeros casos de personas contagiadas por COVID-19, se decreta por el ejecutivo nacional la cuarentena social, colectiva y voluntaria, desde donde entonces se suspendieron las clases presenciales en un primer momento por 30 días, sin embargo, actualmente se mantiene el cierre temporal de las instituciones educativas, y las clases a través del Plan Universidad en Casa continúan bajo la modalidad a distancia.

Ante las actuales circunstancias, la comunidad universitaria representada por estudiantes y docentes de estas casas de estudios han hecho un gran esfuerzo, para adoptar metodologías apoyadas en las Tecnologías de la Información y Comunicación (TIC), de manera que se dé continuidad con la formación académica en estos tiempos de cuarentena. De esta manera, para llevar a cabo el plan Universidad en Casa y dar continuidad a las clases de manera online y cumplir con dicho programa, es necesario que los estudiantes y el personal docente tengan conectividad a internet o por lo menos posean teléfonos con conexión a datos móviles que les permita la comunicación y la retroalimentación en el proceso de enseñanza-aprendizaje.

Ahora bien, de acuerdo a los datos aportados por el Banco Mundial 
(2020): la población de Venezuela alcanza los 28.870 .195 habitantes para el año 2018 , de los cuales 20.731.169 personas poseen teléfonos móviles ( $71.8 \%$ de la población); por otro lado, el número total personas que usan internet en el país está representado por un $72 \%$ de la población para la misma fecha.

Estos datos demuestran que ante esta coyuntura actual y en la manera que se logre salir adelante y volver a la normalidad en las aulas de clases, todas las instituciones universitarias deben aprovechar que una parte de los hogares venezolanos tiene acceso a internet y/o teléfonos móviles, lo que representa una gran oportunidad y excelente potencial para que las instituciones combinen entornos de aprendizaje con un diseño presencial y virtual en el que los procesos de formación estén mediados a través de las TIC (b-learning).

No obstante, sin olvidar la cantidad de estudiantes y/o docentes que no poseen recursos tecnológicos, lo que les impide hacer seguimiento al plan de educación a distancia, es necesario que se tomen acciones, y que se realicen adaptaciones y se ofrezcan diferentes alternativas a la modalidad virtual, de manera de flexibilizar los espacios sin detrimento en los resultados del proceso de enseñanza-aprendizaje.

Por otra parte, el acceso a los servicios públicos en Venezuela en los últimos años se ha agudizado, en especial la crisis eléctrica que limita las condiciones de conectividad, siendo uno de los principales desafíos para cumplir el plan de educación a distancia, sobre todo en las diferentes localidades del Estado Zulia donde se registran largos y frecuentes cortes de electricidad, y consecuencia las constantes fallas en el servicio de internet y datos móviles.

Por consiguiente, es necesario que se planteen soluciones mediante el uso de diversos medios de comunicación con características de multimodalidad, haciendo especial énfasis en herramientas de comunicación 
sincrónicas y asincrónicas; por ejemplo: mensajería de texto, uso de la radio y la televisión, telefonía fija, correo electrónico, mensajería instantánea a través de aplicaciones móviles como WhatsApp o Telegram, redes sociales, TV-Web, hasta espacios con contenidos virtuales en la web. Es importante acotar que la introducción de la multimodalidad en las instituciones universitarias, requiere un programa de formación y actualización docente, así como la vinculación con toda la comunidad académica con el fin de adoptar entornos de aprendizajes b-learning.

Ante todo lo planteado, la intención de este artículo es conocer la situación actual de la implementación del Plan Universidad en Casa adoptado como medida de prevención AntiCOVID-19 en la Universidad Politécnica Territorial del Zulia (UPTZ), con el fin de reflexionar sobre desafíos y oportunidades que enfrentan las universidades ante la virtualización de la enseñanza en tiempos actuales; cuestionando si los docentes y estudiantes disponen recursos o herramientas tecnológicas para enfrentar esta situación, así como la adecuada formación en entornos virtuales para llevar a cabo actividades académicas y de investigación.

\section{Aportes teóricos}

\subsection{Educación a distancia}

La Educación a distancia, es definida por otros autores como educación virtual, educación en línea, formación online, formación a través internet, elearning o educación a través de las TIC. Estos términos en algunas ocasiones y contextos se diferencian, pero para los fines de este artículo se utilizará la educación a distancia para describir la modalidad de educación que está mediada a través de las TIC. Para Juca (2016a), en la educación a distancia:

[...] No existe una relación directa en tiempo real para que el docente dirija el proceso y el proceso de aprendizaje del estudiante es más flexible, no existe coincidencia física en cuanto al lugar y al tiempo, exige mayor independencia y 
OAI-PMH: http://www.indteca.com/ojs/index.php/Revista_Scientific/oai

Artículo Original / Original Article

autorregulación por parte del estudiante. La educación a distancia adopta diversas peculiaridades en función de la intermediación, del tiempo y del canal que se vaya a utilizar (pág. 107).

En relación con lo anterior, la educación a distancia es considerada un modelo de formación no presencial, en el cual los elementos de tiempo, espacio y comunicación no limitan el proceso de enseñanza-aprendizaje; por su parte, en la educación a distancia la formación se da por mediación pedagógica y andragógica, a través del uso de las diferentes TIC.

Afirman Lozano y Giralt (2014a): que la comunicación en espacios virtuales se denomina multimodal, la cual contempla distintos recursos como: la imagen, el texto escrito, la disposición de la información, la música, los gestos, la lengua oral, imágenes en movimiento u objetos 3D, entre otros. En este sentido, la aplicación de la comunicación multimodal está caracterizada por utilizar diversos canales particulares a través de las diferentes tecnologías de información y comunicación como: la radio, la televisión, telefonía fija, telefonía móvil, los dispositivos tecnológicos como el ordenador, tablet, smartphone, el uso de medios a través del internet como los servicios de correo electrónico, chats, videoconferencias, blogs, foros, plataformas tecnológicas, redes sociales, entornos virtuales, canales digitales a través de diferentes opciones como YouTube, Facebook, entre otros.

Dentro del marco de la adopción de la modalidad de educación a distancia, en las instituciones universitarias venezolanas generada por la crisis sanitaria de la Pandemia COVID-19, ha permitido la integración y combinación de diferentes herramientas de comunicación multimodal, con el fin de que los contenidos educativos objeto de aprendizajes estén al alcance, además de permitir el contacto y la retroalimentación de la comunidad académica.

Es importante destacar que, gran parte de los estudiantes y docentes de las instituciones universitarias de hoy en día poseen un teléfono con 
conexión a datos móviles o por lo menos un teléfono de tecnología convencional para mensajería de textos o llamadas, lo que les permite enviar y recibir información en tiempo real. Al combinar estas herramientas en la modalidad a distancia el estudiante puede aclarar dudas e inquietudes que se le presenten durante el proceso de enseñanza-aprendizaje.

Es necesario que docentes y estudiantes posean competencias básicas sobre el uso de las TIC, ya que a través de éstas se establecerá la comunicación, presentación e intercambio de la información referente a los contenidos educativos, recursos bibliográficos, metodología docente y cualquier otro contenido correspondiente al programa de formación académico.

\subsection{Herramientas tecnológicas empleadas en la Educación a distancia}

La educación a distancia es una modalidad de educación apoyada en diversas herramientas y/o tecnologías de la comunicación, la cual en los últimos años ha ganado terreno en los espacios académicos como modalidad alternativa ante los avances y la innovación de las TIC. En esa misma línea, Flores y Garrido (2019a): plantean que las TIC "son catalizadores que transforman y modifican los actuales escenarios de aprendizaje” (pág. 46); el uso de las TICs permitirá "[...] buscar, seleccionar, analizar, depurar, utilizar y comunicar la información que se transformará en conocimiento, propiciando asimilación y consolidación de estos procesos [...]" (pág. 47); de enseñanzaaprendizaje. Dentro de las herramientas tecnológicas empleadas en la educación a distancia, encontramos las herramientas sincrónicas y asincrónicas que se describirán a continuación.

\subsubsection{Herramientas sincrónicas}

De acuerdo con Araújo (2011), citado por Lay, et al. (2019a), las herramientas sincrónicas: son "permiten una comunicación en tiempo real 
entre los participantes. Este tipo de comunicación se da en situaciones tales como cuando se conversa con alguien a través del teléfono, o cuando se participa de una sesión de videoconferencia o chat" (pág. 4). En referencia al tema, Gómez-Camacho y Gómez del Castillo (2017): exponen que "las conversaciones sincrónicas a través de teléfonos inteligentes se han convertido un medio habitual de la comunicación digital en Internet" (pág. 1078).

Dentro de estas herramientas se pueden considerar la mensajería instantánea a través de aplicaciones móviles como el WhatsApp o Telegram, los cuales permiten al usuario comunicarse con una persona o grupos de personas conectadas en internet, a través del cual se puede enviar e intercambiar fotos, videos, notas de voz, compartir información en forma de texto, enlaces de visualización o descarga de información; entre otros.

Hoy en día, estas herramientas son adoptadas tanto en la modalidad presencial como en modalidad a distancia, ya que se crean espacios de interacción grupal entre los estudiantes, asimismo, estas aplicaciones permiten al docente crear ambientes de aprendizajes para la discusión; y guiar al estudiante en la comprensión de los diferentes contenidos educativos que se imparten, ofreciendo una retroalimentación, fortaleciendo el proceso de enseñanza-aprendizaje y permitiendo la construcción social del conocimiento.

\subsubsection{Herramientas asincrónicas}

Paralelamente Araújo (2011), citado por Lay, et al. (2019b): dice que la comunicación asincrónica "[...] se produce entre dos o más personas que pueden o no, encontrarse físicamente ubicadas en diferentes contextos [...]" (pág. 3). En este sentido, Lay, et al. (2019c): también explica que "no es necesario que los participantes coincidan en el mismo tiempo [...] esta comunicación solo se desarrolla en formato escrito (o textual)" (pág. 3). Dentro de estas herramientas encontramos el correo electrónico, foros, blogs, y otros 
portales online permiten la comunicación a través de mensajes escritos; donde

el usuario debe revisar su cuenta para que el mensaje sea descubierto o entregado.

A pesar de ello, existen otros recursos digitales que combinan formas de comunicación sincrónica y asincrónica, entre estas se encuentra YouTube, considerada como una red social, medio de comunicación o sitio web que permite a los usuarios buscar, subir o descargar videos. Para Ramírez (2016a), la herramienta YouTube:

[...] Permite al docente crear su propia biblioteca virtual de videos para conformar una comunidad de aprendizaje en torno a los contenidos seleccionados por el docente. $Y$ al estudiante, crear su propia biblioteca virtual, con videos seleccionados por el mismo o con producciones de su propia autoría [...] (pág. 542).

En el plano educativo, esta herramienta ha sido de gran valor para las aulas de clases ya que ofrecen a la comunidad académica una infinidad de información en forma de videos tutoriales, con diferentes contenidos educativos que sirven de apoyo a las labores desempeñadas en el proceso de enseñanza-aprendizaje.

Existen otras herramientas tecnológicas o aplicaciones online que combinan la comunicación asincrónica o sincrónica, y que permiten plantear modelos de educación a distancia, tales como: Google Classroom, Mil Aulas, Chamilo, Moodle, Edmodo, etc.; plataformas que le permiten al docente gestionar sus cursos académicos, a través de la asignación de actividades, que se comparten con los recursos necesarios, sirven de repositorio de documentos, y permiten para llevar el control de calificaciones de estudiantes. Dentro de este contexto, se encuentran otras herramientas tecnológicas que a raíz de la crisis sanitaria se han vuelto más populares para realizar videoconferencias, como es: Zoom, Jitsi, GoToWebinar, Google Meet, Microsoft Teams, entre otras. 


\section{Metodología}

Esta investigación sigue una metodología de tipo descriptiva, definida por Hernández, Fernández y Baptista (2014): como aquellas investigaciones donde se "[...] busca especificar las propiedades, las características y los perfiles de personas, grupos, comunidades, procesos, objetos o cualquier otro fenómeno que se someta a un análisis" (pág. 92). Por su parte, la población de estudio la conforma la Universidad Politécnica Territorial del Zulia (UPTZ) y la muestra estuvo representada por 27 docentes y 15 estudiantes adscritos al Programa Nacional de Formación en Procesos Químicos (PNFPQ).

El propósito de este artículo es describir la experiencia de implementar la Educación a Distancia en la UPTZ, modalidad adoptada ante la emergencia sanitaria provocada por la pandemia COVID-19; de esta forma, la investigación permitió identificar y analizar las categorías establecidas como las oportunidades, limitaciones y/o desafíos que enfrenta el uso y adopción de las TIC y la postura frente al tránsito de la modalidad presencial hacia la modalidad a distancia, que se vio obligado a tomar en todas las instituciones universitarias debido el estado de emergencia decretado por el Estado.

Por otra parte, para la recopilación de los datos, se contó con información relativa al PNFPQ como: la cantidad de docentes adscritos, las unidades curriculares abiertas en el actual Periodo Anual Académico 2020-1 y la matrícula de estudiantes que se incorporaron al Plan Universidad en Casa.

En ese marco, la investigación sigue el enfoque cualitativo como el cuantitativo; en la cual se utilizaron diversos tipos de instrumentos de medición, como registros observacionales, la revisión documental; se aplicó un instrumento a docentes y estudiantes de los diferentes trayectos de formación en el PNFPQ. El instrumento de evaluación diagnostica consistió de un cuestionario conformado por 20 preguntas agrupadas en dos secciones: datos generales y recursos o herramientas de comunicación, usados para cumplir el Plan Universidad en Casa; la validación se realizó por pares 
académicos, lo que permitió realizar un diagnóstico sobre las perspectivas de los docentes y estudiantes frente al uso de herramientas tecnológicas para llevar a cabo las actividades académicas en el plan.

El instrumento fue diseñado a través de Formularios de Google Forms, el cual fue aplicado a docentes y estudiantes que cursan estudios en el PNFPQ; finalmente este cuestionario fue enviado por correo electrónico a cada uno de los participantes de este estudio y a través de la mensajería instantánea usando la aplicación móvil WhatsApp.

Asimismo, se consultaron textos y artículos de revistas científicas referentes al tema de investigación; esta revisión involucró un periodo de tiempo donde se reflexionó sobre los postulados Juca (2016b); Alfonso y Giralt (2014b); Flores y Garrido (2019b); Ramírez (2016b); entre otros. Finalmente, se recolectaron y registraron los datos obtenidos de los participantes, con el fin de reflexionar sobre la adopción de la modalidad a distancia en las instituciones universitarias, de manera de hacer frente a los desafíos y oportunidades en torno al paso de los programas de modalidad presencial hacia la virtualización de los aprendizajes.

\section{Resultados y análisis}

La Universidad Politécnica Territorial del Zulia (UPTZ) gestiona un total de 12 Programas Nacionales de Formación (PNF) a nivel de pregrado con titulaciones de Técnico Superior Universitario (TSU), Licenciatura e Ingeniería; igualmente se administran dos Programas Nacionales de Formación Avanzada (PNFA) a nivel de Postgrado con titulaciones de Especialización, Maestrías y Doctorados.

Para este estudio se analizó la adopción de la metodología de Educación a distancia en el PNF en Procesos Químicos de la UPTZ, el cual gestiona para el actual Periodo Anual Académico 2020-1, un total de 40 Unidades Curriculares (UC) distribuidas como se indica en la tabla 1. En dicha 
tabla se muestra el número de docentes asignados, así como la matrícula de estudiantes activos o atendidos a través del Plan a distancia, así como los estudiantes inactivos o desatendidos, ya que presentan limitaciones para seguir la programación.

Tabla 1. Distribución UC que adoptaron la Modalidad a Distancia.

\begin{tabular}{|l|c|c|c|c|c|}
\hline \multirow{2}{*}{ Tipo de Unidad Curricular (UC) } & \multirow{2}{*}{$\begin{array}{c}\text { Cantidad } \\
\text { UC }\end{array}$} & \multirow{2}{*}{$\begin{array}{c}\text { Cantidad de } \\
\text { Docentes }\end{array}$} & \multicolumn{3}{|c|}{ Cantidad de Estudiantes } \\
\cline { 4 - 6 } & & & Inscritos & Activos & Inactivos \\
\hline UC Básicas y Transdisciplinarias & 8 & 5 & 196 & 114 & 82 \\
\hline UC Especificas & 15 & 10 & 246 & 156 & 90 \\
\hline UC Investigación e Innovación & 5 & 4 & 93 & 65 & 28 \\
\hline UC Electivas & 2 & 1 & 26 & 22 & 4 \\
\hline UC Sociocríticas & 6 & 4 & 92 & 65 & 27 \\
\hline UC Acreditables & 4 & 3 & 91 & 65 & 26 \\
\hline
\end{tabular}

Fuente: Las Autoras (2020).

Es preciso indicar que 17 de estas unidades curriculares requieren contenidos prácticos, talleres y/o laboratorios, pero en cambio, las mismas se han sumado al Plan a distancia con el fin de adelantar los contenidos teóricos. De igual forma, se prevé establecer una reprogramación para estas unidades curriculares mediante cursos de nivelación, una vez que las autoridades educativas permitan de reinicio de actividades presenciales.

Cabe mencionar que los docentes emiten reportes semanales al coordinador académico del programa, el cual remite a la División Docente sobre: actividades académicas, metodología de evaluación y estudiantes activos e inactivos. En el gráfico 1, podemos observar el porcentaje de estudiantes activos (por encima del 58\%) que han seguido el plan en función de las unidades curriculares, y podemos ver la cantidad de estudiantes inactivos (hasta un $42 \%$ ) que manifiestan no cumplir de manera óptima la modalidad a distancia debido a diversas limitaciones. 


\section{Artículo Original / Original Article}

Gráfico 1. Porcentaje de Estudiantes por unidades curriculares.

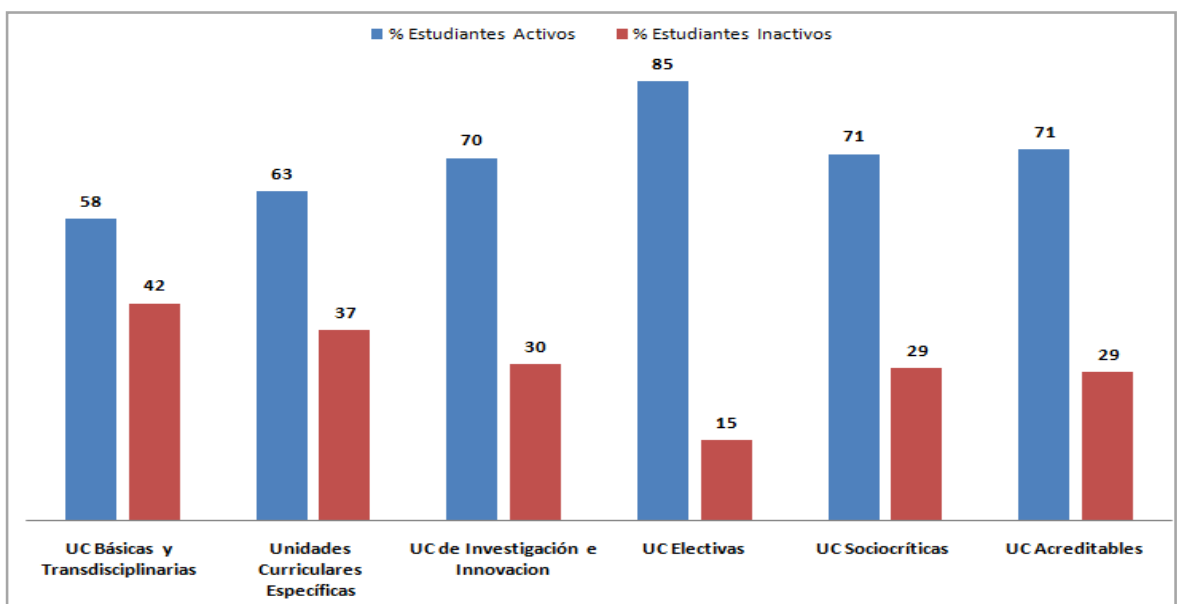

Fuente: Las Autoras (2020).

El mayor desafío que manifiestan los estudiantes y docentes universitarios es luchar con los constantes cortes del servicio eléctrico que limita la conectividad a internet y la calidad de los datos móviles. Para nadie es un secreto que el Estado Zulia es la región más golpeada por la crisis eléctrica desde el año 2019.

En cuanto a los recursos disponibles para llevar a cabo el Plan a distancia, la mayoría de los docentes y estudiantes, manifiestan contar con un computador; como se visualiza en el gráfico 2 , el $70,4 \%$ de los docentes y $60 \%$ de los estudiantes tienen en el hogar al menos un equipo de computación.

Gráfico 2. Cantidad de participantes con equipos de computación.

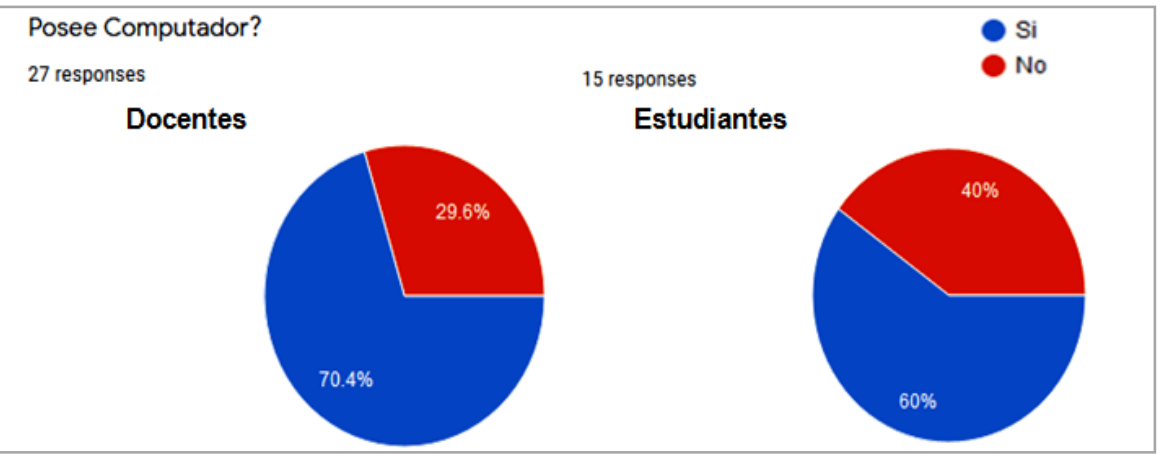

Fuente: Las Autoras (2020). 
La mayoría de los participantes del estudio manifestaron que el computador es de uso familiar, y que deben compartir el equipo con los integrantes del hogar. Esta situación genera estrés adicional aunado a las condiciones de cuarentena, indicando que dos o tres personas en el hogar deben trabajar y estudiar al mismo tiempo con un computador.

En cuanto a la cantidad de hogares con conexión a internet, como se presenta el gráfico 3 , el $63 \%$ de los docentes tienen acceso a internet, en contraposición del $53,3 \%$ de los estudiantes que manifiestan no tener acceso al servicio. Destacando que, los participantes con acceso a internet poseen un servicio intermitente debido a las fallas en el servicio eléctrico, y que deben luchar con los datos móviles para cumplir las actividades académicas.

Gráfico 3. Cantidad de participantes con acceso a internet.

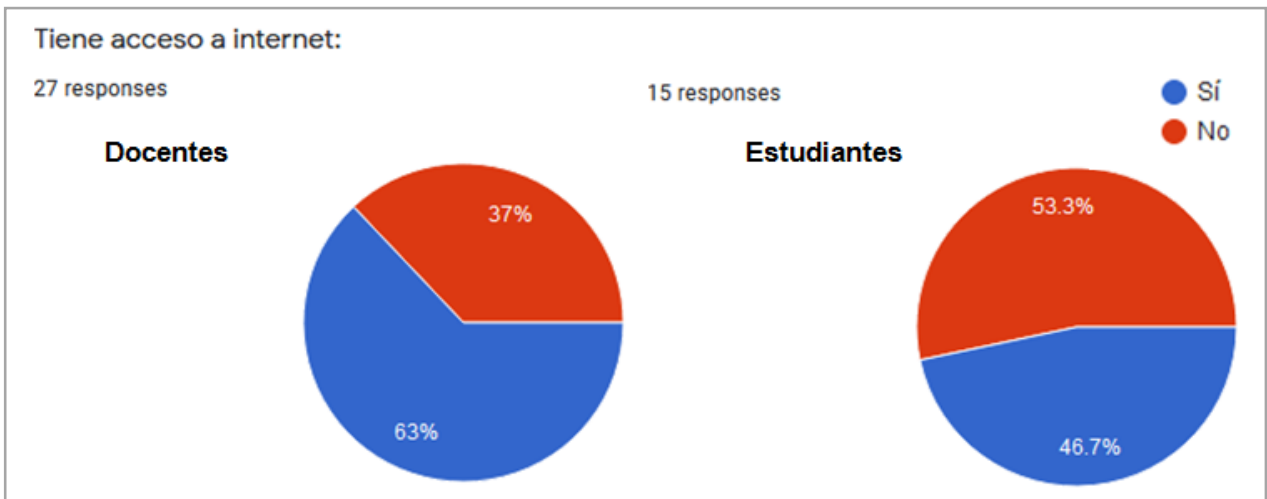

Fuente: Las Autoras (2020).

En cuanto a la cantidad de participantes que disponen de equipos móviles, el total manifiesta contar con un teléfono celular; el 88,9 \% de los docentes y el $93,3 \%$ de los estudiantes poseen equipos propios y un pequeño porcentaje poseen equipos prestados por un integrante del hogar, expresado en el gráfico 4; en función del tipo de teléfono móvil que poseen, la mayoría de los docentes y estudiantes indican que poseen un smartphone. 


\section{Artículo Original / Original Article}

Gráfico 4. Cantidad de participantes con equipos móviles (Celular).

El Teléfono Celular que posee es:

27 responses

Docentes

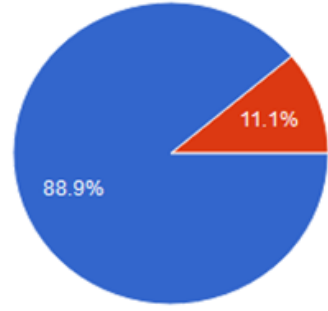

Fuente: Las Autoras (2020)

En cuanto a las herramientas e instrumentos de comunicación utilizados por los docentes y estudiantes para garantizar que se cumpla el Plan de Universidad en Casa, como se puede visualizar en el gráfico 5 y el gráfico 6 , se destacan las siguientes: llamadas telefónicas, mensajería de texto, mensajería instantánea a través de WhatsApp o Telegram, correo electrónico, video llamadas, o aulas virtuales. Precisando que los docentes canalizan el envío de las actividades, contenidos educativos y/o recursos necesarios a través de las distintas herramientas mencionadas anteriormente; posteriormente se realizan evaluaciones individúales o grupales.

Gráfico 5. ¿Qué tipo de comunicación usa frecuentemente con sus estudiantes?.

27 responses

Docentes

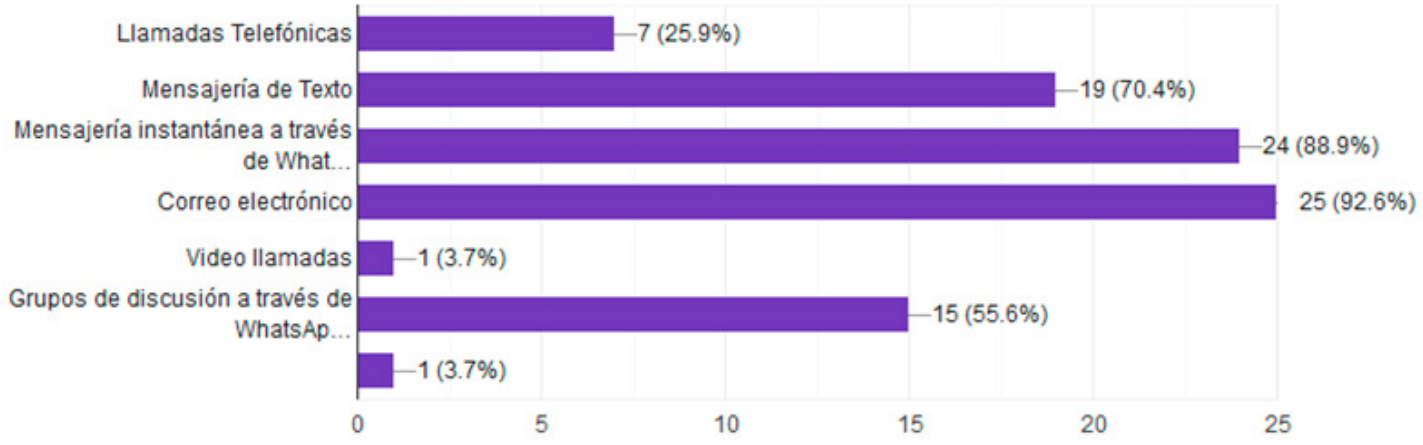

Fuente: Las Autoras (2020). 
Gráfico 6. ¿Qué tipo herramientas usa frecuentemente para cumplir actividades del Plan de Educación a Distancia y estar en comunicación con los profesores?.

15 responses

Estudiantes

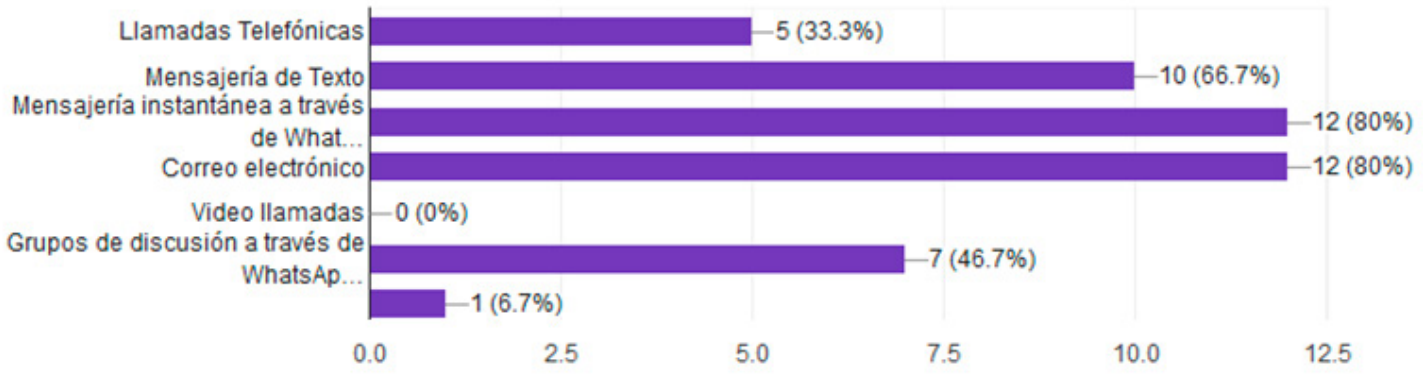

Fuente: Las Autoras (2020).

Es importante destacar, que la mayoría de los docentes a raíz de la implementación de este decidieron abrir grupos con sus estudiantes a través WhatsApp o Telegram, con el fin de establecer discusiones y debates en cuanto a los temas educativos impartidos, para aclarar dudas generadas en el proceso de enseñanza-aprendizaje.

De igual modo, los estudiantes manifiestan que, si hay alguna duda o problema para cumplir las actividades en el Plan a distancia, se soluciona con cualquier herramienta de comunicación, ya sea sincrónica a través de una llamada telefónica o mensajería instantánea con el docente, o asincrónica a través de un correo electrónico, con el fin de hacer contacto con el docente para aclarar inconvenientes suscitados en el proceso.

Por otro lado, parte de los docentes afirman usar aulas virtuales a través de plataformas online como Google Classroom o Moodle; la mayoría plantea no tener formación en estas herramientas. Del mismo modo, un pequeño porcentaje alrededor de $1 \%$ de los docentes manifiesta usar video llamadas o videoconferencias a través de aplicaciones móviles como Jitsi o Zoom.

Es preciso indicar que, depende del tipo de comunicación que desee el 
docente con sus estudiantes, ya sea escrita, o centradas en el audio y video, se ve limitada por la conectividad virtual o señal telefónica; a pesar de los inconvenientes que se presentan, gran parte de los docentes se han adaptado a la modalidad a distancia, realizando grandes esfuerzos para llevar a cabo las actividades de formación con sus estudiantes.

En cambio, todos los participantes del estudio manifestaron que antes de decretarse la cuarentena social combinaban en clases presenciales el uso herramientas tecnologías como: chat, mensajería instantánea, correo electrónico, entre otros; con el fin de mejorar el proceso de enseñanzaaprendizaje con sus estudiantes.

En base a los resultados obtenidos a partir de las respuestas y opiniones generadas por los docentes y estudiantes, se exhibe en el cuadro 1, el resumen de las oportunidades y desafíos enfrentados ante la adopción forzada de la modalidad a distancia, como medida de prosecución de estudios universitarios en la época de cuarentena.

Cuadro 1. Oportunidades y Desafíos del Plan de Educación a distancia.

\begin{tabular}{|c|c|}
\hline \multicolumn{2}{|r|}{ PLAN DE EDUCACIÓN A DISTANCIA } \\
\hline OPORTUNIDADES & $\begin{array}{l}\text { - La mayoría de los docentes y estudiantes tienen acceso a un equipo de } \\
\text { - Ante la situación de la cuarentena social existe ánimo, voluntad, capacidad } \\
\text { y cooperación entre los docentes, mejorando y estableciendo las } \\
\text { relaciones e interacciones con sus estudiantes. } \\
\text { - La educación a distancia permite combinar una gran variedad de } \\
\text { herramientas e instrumentos de comunicación multimodales, permitiendo } \\
\text { superar barreras de tiempo y espacio; logrando la inmediatez } \\
\text { comunicativa. } \\
\text { - Posibilidad de utilizar la experiencia de haber tomado la modalidad a } \\
\text { distancia debido a la cuarentena, y poner en práctica la modalidad b- } \\
\text { learning como metodología para mejorar los entornos de aprendizajes } \\
\text { post-pandemia. } \\
\text { - Accesibilidad y mayor volumen de información, así como, metodologías y } \\
\text { recursos didácticos disponibles a través de la web. } \\
\text { Esta modalidad permite ofrecer una mejor retroalimentación e interacción } \\
\text { docente-estudiante, obteniendo alta producción e intercambio de } \\
\text { conocimientos. }\end{array}$ \\
\hline DESAFÍOS & $\begin{array}{l}\text { - Constantes cortes y/o fallas eléctricas de la región, lo que dificulta la } \\
\text { conectividad virtual. } \\
\text { - La modalidad a distancia se ve afectada por el poder adquisitivo de los } \\
\text { docentes y estudiantes, debido a los altos costos en los servicios de } \\
\text { internet y/o datos móviles, así como los recursos tecnológicos limitan el }\end{array}$ \\
\hline
\end{tabular}


proceso de enseñanza-aprendizaje.

- La mayoría de los docentes manifiestan no tener formación en entornos y plataformas virtuales, por lo que se requiere un plan de actualización permanente del docente y de la comunidad académica, para el uso, manejo de las TIC y aulas virtuales, con el fin de impartir clases bajo la modalidad a distancia.

- Diseño y actualización constante de plataformas web universitarias.

- La obsolescencia de las tecnologías.

\section{Reflexiones Finales}

La virtualización forzada a la que se vieron sometidas las instituciones venezolanas debido a las medidas de confinamiento ante la pandemia COVID19 , surge como una oportunidad para adoptar herramientas digitales en los espacios educativos; es necesario llevar a cabo un rediseño curricular al método presencial y asumir nuevas formas de enseñanza y aprendizaje, considerándola una alternativa de calidad inspirada a través de las TIC.

Por su parte, el retorno de las actividades presenciales en las universidades dependerá de las autoridades sanitarias sobre el avance de la pandemia, en sí sabemos que el Plan Universidad en Casa se mantendrá hasta Julio. Por lo que, esta situación está generando una profunda reflexión sobre los diversos modelos de transferencia de conocimientos y la introducción de las tecnologías en las universidades.

Por su parte, las posibilidades de implantar la modalidad de educación a distancia continúan pese a las limitaciones de conectividad virtual, la crisis eléctrica, la inexistencia o falta de actualización de infraestructuras y plataformas web universitarias, la falta de formación de la comunidad académica en competencias digitales, entre otros aspectos. Bajo estas circunstancias, es necesario que se tomen acciones con el fin de atender a la población docente y estudiantil que carecen de recursos tecnológicos, así como los que no tienen acceso al Internet. Pese a la situación, se reconoce el gran esfuerzo de adaptación y corresponsabilidad de la comunidad académica 
para llevar a cabo las actividades bajo la modalidad a distancia.

Incluso, sin dejar atrás las funciones de extensión de las universidades, es importante que se tomen en cuenta actividades apoyado en las TIC, donde se propicien espacios de interacción entre estudiantes y docentes manteniendo las medidas preventivas y el distanciamiento social, con el fin de fortalecer el feedback en el proceso de enseñanza-aprendizaje.

\section{Referencias}

Banco Mundial (2020). Personas que usan Internet (\% de la población). Washington, Estados Unidos: Grupo Banco Mundial. Recuperado de: https://datos.bancomundial.org/indicador/IT.NET.USER.ZS

Flores, D., \& Garrido, J. (2019). Competencias digitales para los nuevos escenarios de aprendizaje en el contexto universitario. Revista Scientific, 4(14), 44-61, e-ISSN: 2542-2987. Recuperado de: https://doi.org/10.29394/Scientific.issn.2542-2987.2019.4.14.2.44-61

Gómez-Camacho, A., \& Gómez del Castillo, M. (2017). La Norma Escrita en las conversaciones de WhatsApp de estudiantes universitarios de posgrado. Rmie, 22(75), 1077-1094, e-ISSN: 1405-6666. Recuperado de: http://www.scielo.org.mx/pdf/rmie/v22n75/1405-6666-rmie-22-7501077.pdf

Hernández, R., Fernández, C., \& Baptista, M. (2014). Metodología de la Investigación. Sexta edición, ISBN: 978-1-4562-2396-0. México: McGraw-Hill / Interamericana Editores, S.A. de C.V.

Juca, F. (2016). La educación a distancia, una necesidad para la formación de los profesionales. Universidad Y Sociedad, 8(1), 106111, e-ISSN: 2218-3620. Recuperado de:

https://rus.ucf.edu.cu/index.php/rus/article/view/312

Lay, N., Márceles, V., Parra, M., Pirela, A., De Castro, N., Yarzagaray, J., ... Ramírez, J. (2019a,b). Uso de las herramientas de comunicación 
asincrónicas y sincrónicas en la banca privada del municipio Maracaibo (Venezuela). Espacios, 40(4), 1-9, e-ISSN: 0798-1015. Recuperado de:

https://www.revistaespacios.com/a19v40n04/19400411.html Lozano, R., \& Giralt, M. (2014a,b). El porfolio digital y la comunicación multimodal en el aprendizaje de la lengua oral: estudio piloto de un caso. En L. P. Cancelas, R. Jiménez-Fernández, M. F. Romero Oliva y S. Sánchez Rodríguez (Coords.). Una educación lingüística y literaria en el siglo XXI (1-11). Granada: Grupo Editorial Universitario.

Ramírez, M. (2016a,b). Posibilidades del Uso Educativo de YouTube. Ra Ximhai, 12(6), 537-546, e-ISSN: 1665-0441. Recuperado de: http://www.revistas.unam.mx/index.php/rxm/article/view/71911/63438 


\section{Eddymar María Flores Nessi}

e-mail: eddymarf.nessi@gmail.com

Nacida en la ciudad de Cabimas, Estado Zulia, Venezuela,

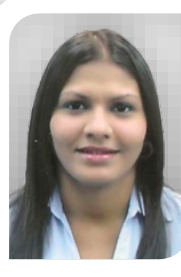
el 11 de marzo del año 1984. Ingeniera Química por la Universidad del Zulia (LUZ, 2007); Magister en Gerencia de Proyectos de Investigación y Desarrollo en la Universidad Dr. Rafael Belloso Chacín (URBE, 2016); con experiencia docente a nivel universitario desde hace 10 años; actualmente Docente a Tiempo Completo en la Universidad Politécnica Territorial del Zulia (UPTZ); Docente de las Unidades Curriculares: Proyectos, Ingeniería de las Reacciones Químicas, Ingeniera Ambiental; Tutora y jurado evaluador de trabajos y proyectos de Investigación; Experiencia en la asesoría y consultoría ambiental referida a la elaboración de estudios de impacto ambiental, diagnósticos ambientales y tramitación de autorizaciones ambientales. 
OAI-PMH: http://www.indteca.com/ojs/index.php/Revista_Scientific/oai

Artículo Original / Original Article

Jenny Macarena Meléndez Mora

e-mail: immelendezm@gmail.com

Nacida en la ciudad de Bachaquero, estado Zulia,

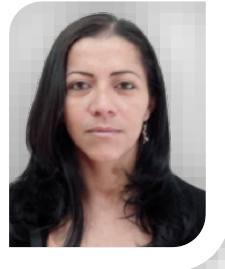
Venezuela, el 7 de mayo del año 1977. Máster en Geofísica Aplicada, Mención búsqueda de Petróleo y Gas de la Universidad Tecnológica de la Habana "José Antonio Echeverría", Convenio Cuba-Venezuela (CUJAE, 2015); Ingeniero de Petróleo de la Universidad del Zulia (LUZ, 2008); Técnico Superior Universitario en Hidrocarburos Mención Petróleo del Instituto Universitario de Tecnología de Cabimas (IUTC, 2001); con experiencia docente desde hace más de 17 años; actualmente Docente en la categoría de Agregado a Dedicación Exclusiva en la Universidad Politécnica Territorial del Zulia (UPTZ); Tutora y jurado evaluador de trabajos y proyectos de Investigación del Departamento de Hidrocarburos. 


\section{María Alejandra Baptista Montero}

e-mail: marialebap@gmail.com

Nacida en la ciudad de Cabimas, Estado Zulia, Venezuela,

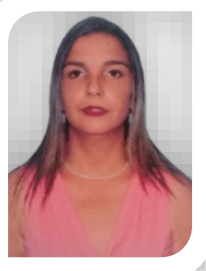

el 15 de enero del año 1979. Máster en Geología, Mención

Exploración y Producción de Yacimientos de Petróleo y

Gas del Instituto Superior Minero Metalúrgico de Moa "Dr.

Antonio Núñez Jiménez", Convenio Cuba-Venezuela

(ISMMANJ, 2015); Ingeniero de Petróleo de la Universidad

del Zulia (LUZ, 2005); con experiencia docente en la Universidad Bolivariana de Venezuela desde el año 2007 hasta el año 2019; Tutora y Jurado Evaluador de trabajos y proyectos de investigación de los Programas de Formación de Grado en Petróleo, Gas, Refinación y Petroquímica; actualmente, me desempeño como Analista de Diseño y Planificación Educativa; y facilitadora en la Gerencia de Gestión Humana de la empresa Petroquímica de Venezuela, S.A. desde el año 2019.

El contenido de este manuscrito se difunde bajo una Licencia de Creative Commons ReconocimientoNoComercial-Compartirlgual 4.0 Internacional 